\title{
Disclosing molecules life: what would you do if you were a hormone?
}

Azevedo- Martins, A.K. and Nunes, V.A.

Escola de Artes, Ciências e Humanidades, EACH-USP, São Paulo, SP, Brazil

Teaching Biochemistry and Molecular Biology commonly requires great efforts of teachers and students in order to facilitate the apprehension of complexes and theoretical subjects. The contemporist approaches have aimed the development of thinking skills with different levels of complexity such as knowledge, application, synthesis and creation. In this context, drama has emerged as an educational strategy to help students to disclosure and learn about molecular events, whereas allows them to reflect about themselves and the learning process. This work aimed to promote an original scenario to improve learning about hormones action mechanisms, which is part of the Biological Basis of Gerontology program (EACH-USP). Students were invited to take part in short plays involving hormones action such as activation of hypothalamus-hypofisis-adrenal axis in stress, postprandial glicemia regulation, menstrual cycle and fecundation. The main requirement was that students should act as molecules. The following aspects of play making were evaluated: researching, script writing, accurate information, creativity and relationship between performance and biochemical characteristics of molecules. Evaluation of this activity is very positive since several pedagogic aims were achieved such as better comprehension about hormones action and their effects, precise localization of the events and its correlation with others systems, and application of the endocrinology content to everyday situations, that indicate knowledge appropriation in different dimensions. Moreover, we found consistency of our observations with students reports about their experience. 